\title{
Contaminación del agua en fuentes cercanas a campos petrolíferos de Bolivia
}

\author{
Silvia González Alonso, ${ }^{1}$ Jesús Esteban-Hernández, ${ }^{1}$ \\ Yolanda Valcárcel Rivera, ${ }^{1}$ Valentín Hernández-Barrera ${ }^{1}$ \\ y Ángel Gil de Miguel ${ }^{1}$
}

Forma de citar González Alonso S, Esteban-Hernández J, Valcárcel Rivera Y, Hernández-Barrera V, Gil de Miguel A. Contaminación del agua en fuentes cercanas a campos petrolíferos de Bolivia. Rev Panam Salud Publica. 2010;28(4):235-43.

RESUMEN Objetivo. Determinar las concentraciones de compuestos petroquímicos en las fuentes de agua de consumo para comunidades cercanas a campos petroliferos del Chaco Boliviano.

Métodos. Se recogieron datos sobre concentraciones de hidrocarburos totales de petróleo (HTP), 16 hidrocarburos aromáticos policíclicos (HAP), incluidos el benceno, tolueno, etilbenceno y xilenos (BTEX), y 22 metales en muestras de 42 fuentes de agua de consumo humano situadas a menos de $30 \mathrm{~km}$ de un campo de extracción de petróleo. Se analizó la distribución de la concentración y el cumplimiento de los estándares definidos en las normativas boliviana, europea y estadounidense, así como en las recomendaciones de la Organización Mundial de la Salud.

Resultados. En $76,19 \%$ de las muestras se halló algún contaminante petroquímico en concentraciones superiores a alguna de las cuatro normativas de referencia. Las muestras de agua que presentaron mayor contaminación fueron las provenientes de grifos y ríos. Los contaminantes más frecuentes fueron HTP, HAP, aluminio, arsénico, manganeso y hierro.

Conclusiones. Las comunidades del Chaco Boliviano ubicadas en un radio de $30 \mathrm{~km}$ alrededor de los campos de extracción de petróleo consumen agua con concentraciones de HTP, HAP y metales muy por encima de los niveles permitidos por la normativa boliviana y los estándares internacionales, poniendo en grave riesgo la salud pública de sus habitantes.

Palabras clave Contaminación del agua; contaminantes del agua; fuentes públicas de agua; agua potable; contaminación química; contaminación de ríos; hidrocarburos; petróleo; salud ambiental; Bolivia.

La extracción de petróleo es una de las principales actividades económicas y la mayor fuente de inversión extranjera de Bolivia (1). El petróleo se suele definir como una mezcla compleja de parafinas, nafténicos e hidrocarburos aromáticos,

\footnotetext{
Universidad Rey Juan Carlos, Facultad de Ciencias de la Salud, Departamento de Medicina Preventiva y Salud Pública e Inmunología y Microbiología Médica. Madrid, España. La correspondencia se debe dirigir a Silvia González Alonso, silvia.alonso@urjc.es
}

incluido el benceno, y una pequeña cantidad de sulfuro, nitrógeno, oxígeno y compuestos metálicos (2). Sus componentes químicos potencialmente perjudiciales para la salud son múltiples. Los hidrocarburos aromáticos policíclicos (HAP) en conjunto son considerados muy tóxicos para el ser humano y muchos de ellos son clasificados como cancerígenos, mutagénicos o teratogénicos (3).

Hasta la fecha, los estudios sobre el tema se han enfocado sobre todo en los efectos que tiene la exposición ocupacional a los componentes del petróleo y a los riesgos sanitarios que entrañan los vertidos, fundamentalmente marinos. En todo caso, los menos frecuentes son los trabajos epidemiológicos realizados en comunidades cercanas a zonas de actividades petroquímicas. Estos estudios muestran que esas poblaciones corren un mayor riesgo de presentar alteraciones cutáneas y complicaciones respiratorias, enfermedades neoplásicas, tumores 
cerebrales, melanoma y cáncer de pulmón (4-13). También han detectado un aumento en la incidencia de nacimientos prematuros, abortos espontáneos y bajo peso al nacer (14-18).

Las empresas petroleras, sin embargo, no han mostrado mucha preocupación por las huellas que sus actividades dejan en el medio natural, cultural y socioeconómico de los lugares donde operan o han operado (19). A este comportamiento se suma la deficiente vigilancia gubernamental para verificar el cumplimiento de los compromisos adquiridos por estas empresas para garantizar la salud y el bienestar de las poblaciones cercanas a sus actividades petroquímicas (20).

En Bolivia, la construcción de gasoductos, los derrames de petróleo y las actuaciones de petroleras en el Chapare han traído aparejadas agresiones medioambientales, incluida la contaminación de recursos hídricos, denunciadas por varias organizaciones sociales desde la década de los sesenta (21-24). Si bien hay estudios que prueban la contaminación de recursos hídricos bolivianos con hidrocarburos totales de petróleo (HTP), todavía no hay muchos datos probatorios acerca de su impacto en la salud de las poblaciones afectadas (25-27).

Los ríos son la principal fuente de consumo directo de agua para $27,65 \%$ de la población rural del país, y alimentan los sistemas de agua canalizada existentes (28). El tratamiento del agua que se realiza en los hogares tiene como prioridad mejorar la calidad microbiológica, por lo que se utilizan métodos pasivos de desinfección y cloración residual de bajo costo. Estos tipos de tratamientos pueden ser efectivos para eliminar patógenos, pero no lo son cuando se trata de compuestos químicos como los hidrocarburos o los metales. Más aún, los estudios sobre la mala calidad del agua y las enfermedades por lo general se han dedicado al efecto de la potabilización inadecuada en la aparición de brotes diarreicos a corto plazo, siendo pocos los trabajos orientados a caracterizar el grado de exposición y el impacto sobre la salud de la exposición crónica a otro tipo de compuestos potencialmente transportados por el agua -y raramente eliminados en los procesos convencionales de purificación (29).

El presente estudio se propone determinar las concentraciones de hidrocarburos totales de petróleo, hidrocarburos aromáticos policíclicos, benceno, to- lueno, etilbenceno, xilenos y metales en las fuentes de agua para beber de comunidades situadas a menos de $30 \mathrm{~km}$ de un campo petrolífero en la región boliviana del Chaco.

\section{MATERIALES Y MÉTODOS}

\section{Muestreo}

La selección del Chaco como lugar del estudio se debió a que es la región donde se localizan las mayores reservas de gas y los campos petrolíferos más extensos y productivos del país (30). La elección de las áreas específicas de estudio se realizó mediante un muestreo bietápico por conglomerados, cuya primera etapa correspondió a los campos de extracción de petróleo que cumplían los siguientes requisitos: 1) eran operativos en el momento del estudio, 2) estaban situados a más de $30 \mathrm{~km}$ de cualquier zona de extracción minera o actividad industrial y 3) pertenecían a los departamentos de Chuquisaca, Santa Cruz o Tarija. Los campos de difícil acceso fueron excluidos. La segunda etapa correspondió a la elección de las comunidades, que a su vez debían cumplir con una serie de criterios: 1) tamaño poblacional mayor a 500 individuos adultos, 2) homogéneas en cuanto al número de habitantes (diferencias inferiores a $10 \%$ con respecto a la media de tamaño poblacional) y 3) situadas a una distancia superior de $30 \mathrm{~km}$ de una zona de extracción minera o industrial. También se excluyeron aquellas de difícil acceso. Las comunidades fueron seleccionadas mediante muestreo por conglomerados, proporcional al número de campos de extracción petrolífera cercanos. Finalmente fueron seleccionadas 21 comunidades.

Se recogieron muestras de todas y cada una de las fuentes de agua, utilizadas o potencialmente utilizables por los residentes de cada comunidad, para consumo humano. Se consideró "agua de consumo humano" aquella utilizada para beber, cocinar, preparar alimentos o higiene personal. Las fuentes analizadas incluyeron quebradas, ríos o arroyos, grifos, atajaderos, pozos y lagunas (figura 1). La toma de muestras se llevó a cabo durante septiembre de 2005 —estación seca.

La recogida, procesamiento y conservación de las muestras se realizó siguiendo la norma ISO 5667 (31-33). Brevemente, tras aclarar la botella tres veces con agua de la fuente a analizar, se reco- gieron manualmente $250 \mathrm{~mL}$ de agua, con tres réplicas por muestra, en recipientes de vidrio borosilicatado ámbar. Las muestras fueron estabilizadas con $400 \mu \mathrm{L}$ de $\mathrm{HCl} 6 \mathrm{~N}$ si estaban destinadas a la determinación de hidrocarburos, o con $40 \mu \mathrm{L}$ de $\mathrm{HNO}_{3}$ concentrado al $65 \%$ de pureza si estaban destinadas a la cuantificación de metales $(33,34)$. Se prepararon tres blancos de campo por duplicado. El primer blanco se construyó con agua destilada milli-RO. Siguiendo los procedimientos estándar de los laboratorios externos encargados de realizar las determinaciones, se preparó un segundo blanco mezclando $250 \mathrm{~mL}$ de agua destilada milli-RO con $400 \mu \mathrm{L}$ de $\mathrm{HCl}$ 6N. Por último, se preparó un tercer blanco mezclando $250 \mathrm{~mL}$ de agua destilada milli-RO y $40 \mu \mathrm{L}$ de $\mathrm{HNO}_{3}$ concentrado al $65 \%$ de pureza. Además, se recogieron por triplicado dos muestras escogidas al azar, de fuentes distintas. Los blancos de campo estuvieron sometidos a las mismas condiciones de temperatura $\left(2-5^{\circ} \mathrm{C}\right)$ que el resto de las muestras y se utilizaron para comprobar que los envases y los estabilizantes no introducían errores durante la cuantificación. Las muestras y los blancos fueron enviados a laboratorios externos, que fueron ciegos a su condición durante todo el análisis y, por lo tanto, se pudo validar sus resultados (34). La toma de muestras fue autorizada por los ayuntamientos de las comunidades incluidas en el estudio.

\section{Análisis de las muestras}

La concentración de HTP se determinó mediante espectroscopía infrarroja (FTIR), según la norma de referencia no. 418.1 de la Agencia para la Protección del Medio Ambiente (EPA, por sus siglas en inglés). Los 16 HAP incluidos en la lista de contaminantes prioritarios de la EPA se cuantificaron mediante cromatografía de gases con detector de llama (GC-FID) según la norma EPA-8100. Las concentraciones de los hidrocarburos benceno, tolueno, etilbenceno y xileno (BTEX) se determinaron mediante cromatografía de gases con detector de masas (GC-MS), según la norma EPA8020. En todas las muestras se realizó un análisis de 22 metales: aluminio, arsénico, bario, calcio, cadmio, cromo, cobalto, cobre, estroncio, hierro, litio, magnesio, manganeso, molibdeno, níquel, plomo, potasio, selenio, sodio, titanio, 
FIGURA 1. Localización de las zonas de toma de muestras en las 21 comunidades del estudio sobre contaminación del agua en fuentes para consumo cercanas a campos petrolíferos, Bolivia, 2005

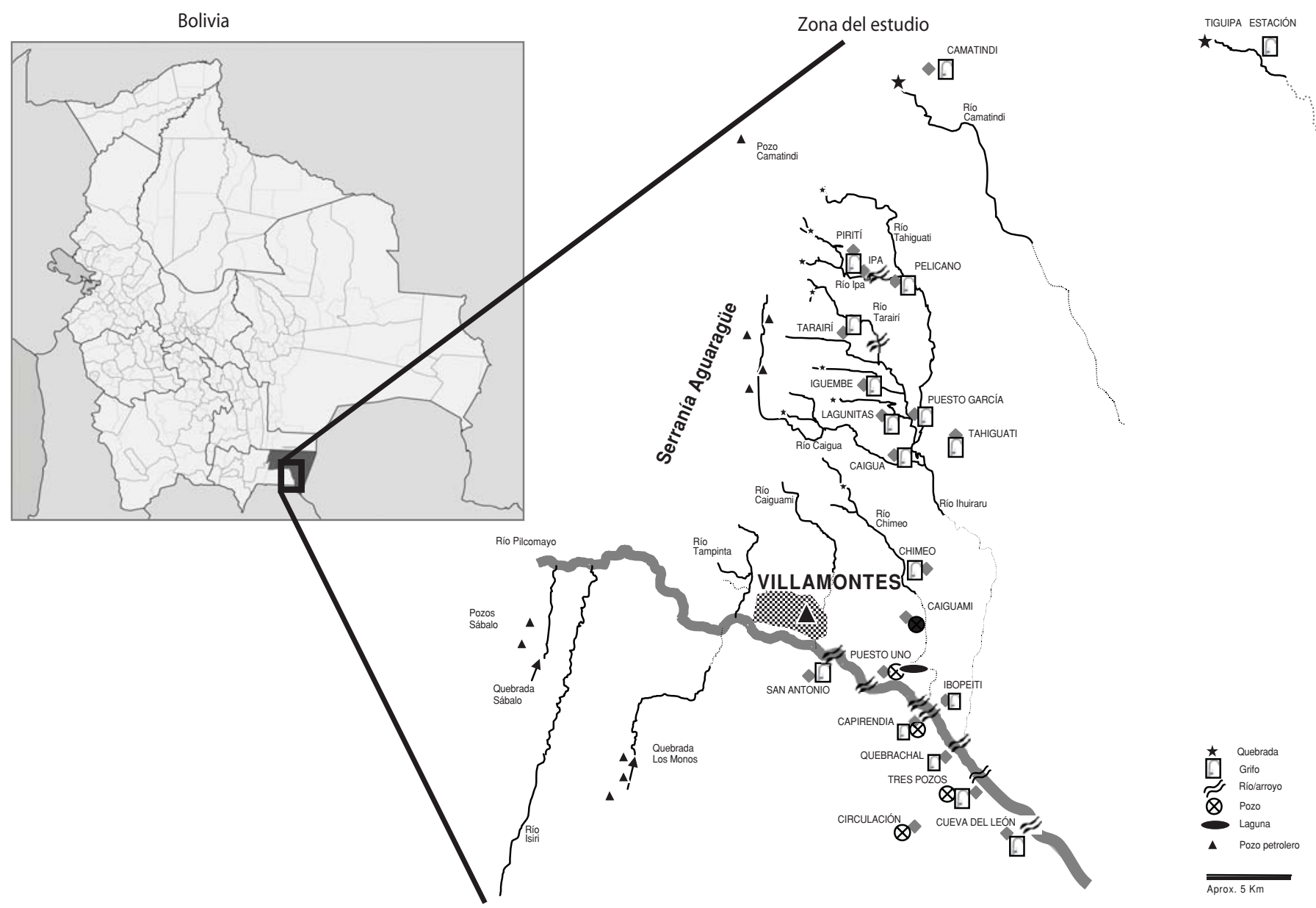

Fuente: elaboración de los autores a partir de mapas aportados por la Municipalidad de Villamontes, Bolivia.

vanadio y zinc. La determinación de metales se realizó mediante espectrometría de emisión atómica en plasma de acoplamiento inductivo (ICP-AES), método $3030 \mathrm{H}(34)$.

\section{Análisis de los datos y marco normativo}

La concentración de los diferentes contaminantes se representa mediante medidas de tendencia central y dispersión. Con el objetivo de describir y comparar el grado de contaminación de las aguas analizadas en función de distintas normativas de referencia, las concentraciones halladas fueron comparadas con los niveles máximos aceptados (valores límite) establecidos en la norma boliviana (N512), en la directiva europea 98/83/CE, en los estándares de la EPA y en las recomendaciones de la Organización Mundial de la Salud (OMS) sobre la calidad del agua potable para consumo humano (cuadro 1) (35-38). En los análisis posteriores, se consideró "muestra contaminada" aquella que contenía alguno de los contaminantes analizados en una concentración superior al límite establecido por la normativa de referencia.

\section{RESULTADOS}

En total se recogieron 42 muestras discretas y puntuales de todas las fuentes de consumo de agua en las 21 comunidades del estudio. Diecinueve muestras correspondieron a agua de río o arroyo (10 de estas en zonas de quebrada), 17 a grifo, 5 a pozo y 1 a laguna. En el cuadro 2 se pueden observar la mediana y el rango de concentración (mínimo y máximo) de los diferentes contaminantes analizados, con el valor máximo admitido por la norma boliviana. La mediana y el rango de la suma de concentraciones de los 16 HAP analizados fueron 4,05 $(0,20-2$ 989) $\mu \mathrm{g} / \mathrm{L}$.

Como se puede ver en la figura 2, el número de muestras contaminadas varía según las normas aplicadas, cuyas diferencias derivan fundamentalmente del número de compuestos contemplados y de los valores aceptados por cada una de ellas. En este sentido, la norma boliviana es la que contempla mayor número de compuestos analizados en el agua de consumo humano $y$, contrariamente, la de Estados Unidos la que reconoce un menor número de compuestos.

Considerando los valores límite más restrictivos de las cuatro normativas utilizadas, 32 de las 42 muestras estaban contaminadas por alguno de los compuestos contemplados. En la figura 3 se compara la proporción de muestras contaminadas en función del contaminante analizado y la fuente de agua de la muestra para las cuatro normativas. 
CUADRO 1. Valores máximos aceptados en el agua para consumo humano de cada uno de los contaminantes analizados en el presente estudio, según las normativas boliviana, europea y estadounidense, así como los recomendados por la OMS, Bolivia, 2005

\begin{tabular}{|c|c|c|c|c|}
\hline \multirow[b]{2}{*}{ Contaminante } & \multicolumn{4}{|c|}{ Valores máximos aceptados ( $\mu \mathrm{g} / \mathrm{L})$} \\
\hline & $\begin{array}{l}\text { Legislación } \\
\text { boliviana }\end{array}$ & $\begin{array}{l}\text { Legislación } \\
\text { europea }\end{array}$ & $\begin{array}{c}\text { Estándares } \\
\text { EPA }\end{array}$ & $\begin{array}{c}\text { Recomendaciones } \\
\text { de la OMS }\end{array}$ \\
\hline Hidrocarburos totales de petróleo & 10 & NA & NA & NA \\
\hline Benceno & 2 & 1 & 5 & 10 \\
\hline Etilbenceno & 300 & NA & 700 & 300 \\
\hline Xilenos (total) & 500 & NA & 10000 & 500 \\
\hline Tolueno & 700 & NA & 1000 & 700 \\
\hline Naftaleno & NA & NA & NA & NA \\
\hline Acenaftileno & NA & NA & NA & NA \\
\hline Acenafteno & NA & NA & NA & NA \\
\hline Fluoreno & NA & NA & NA & NA \\
\hline Fenantreno & NA & NA & NA & NA \\
\hline Antraceno & NA & NA & NA & NA \\
\hline Fluoranteno & NA & NA & NA & NA \\
\hline Pireno & NA & NA & NA & NA \\
\hline Benzo(a)antraceno & NA & NA & NA & NA \\
\hline Criseno & NA & NA & NA & NA \\
\hline Benzo(b)fluoranteno & NA & NA & NA & NA \\
\hline Benzo(k)fluoranteno & NA & NA & NA & NA \\
\hline Benzo(a)pireno & 0,2 & 0,01 & 0,2 & 0,7 \\
\hline Indeno (1,2,3-c-d)pireno & NA & NA & NA & NA \\
\hline diBenzo(a-h)antraceno & NA & NA & NA & NA \\
\hline Benzo(ghi)perileno & NA & NA & NA & NA \\
\hline HAP total (Borneff) & NA & 0,11 & NA & NA \\
\hline Aluminio & 100 & 200 & $50-200$ & 200 \\
\hline Arsénico & 10 & 10 & 10 & 10 \\
\hline Bario & 700 & NA & 2000 & 700 \\
\hline Calcio & 200000 & NA & NA & NA \\
\hline Cadmio & 5 & 5 & 5 & 3 \\
\hline Cobalto & NA & NA & NA & NA \\
\hline Cromo & 50 & 50 & 100 & 50 \\
\hline Cobre & 1000 & 2000 & 1300 & 2000 \\
\hline Estroncio & NA & NA & NA & NA \\
\hline Hierro & 300 & 200 & 300 & 300 \\
\hline Litio & 2500 & NA & NA & NA \\
\hline Magnesio & 150000 & NA & NA & NA \\
\hline Manganeso & 100 & 50 & 50 & 400 \\
\hline Molibdeno & NA & NA & NA & 70 \\
\hline Níquel & 50 & 20 & NA & 20 \\
\hline Plomo & 10 & 10 & 15 & 10 \\
\hline Potasio & NA & NA & NA & $N A^{a}$ \\
\hline Selenio & 10 & 10 & 50 & 10 \\
\hline Sodio & 200000 & 200000 & NA & 200000 \\
\hline Titanio & NA & NA & NA & NA \\
\hline Vanadio & NA & NA & NA & NA \\
\hline Zinc & 5000 & NA & 5000 & 3000 \\
\hline
\end{tabular}

Fuente: elaborado con base en las referencias 35 a 38.

Nota: Abreviaturas. EPA: Agencia para la Protección del Medio Ambiente (Estados Unidos); OMS: Organización Mundial de la Salud; NA: datos no aplicables (no contemplado en la normativa); HAP: hidrocarburos aromáticos policíclicos. a En revisión.

Veintiuna de las muestras tuvieron presencia de HTP por encima de lo permitido por la norma boliviana y 15 registraron contaminación por HAP según la normativa europea. Los metales contaminantes más frecuentes fueron aluminio, arsénico, cromo, hierro, manganeso y níquel, hallados por encima de los valores límite entre 4 y 11 de las muestras analizadas según la normativa utilizada. Grifo y río fueron las fuentes con mayor proporción de muestras contaminadas.
Al aplicar únicamente la norma boliviana, 30 de las 42 muestras estuvieron contaminadas por alguno de los contaminantes considerados en el estudio -5 muestras de agua de quebrada, 12 de grifo, 8 de río, 4 de pozo y 1 de laguna. Los contaminantes que más a menudo se encontraron por encima de los valores aceptados en dicha normativa fueron HTP, presentes en 21 muestras, manganeso en 10, y aluminio, arsénico y hierro en 9. La muestra contaminada por mayor número de compuestos fue la del río de la comunidad de Quebrachal, en la que se detectaron HTP, aluminio, arsénico, cromo, hierro y manganeso por encima de los valores límite. También destacó la muestra de grifo de Lagunitas, que presentó elevadas concentraciones de metales.

\section{DISCUSIÓN}

Los análisis realizados en las fuentes de agua de consumo de las comunidades cercanas $(<30 \mathrm{~km})$ a campos petrolíferos revelaron altas concentraciones de HTP, HAP y metales. No obstante, las normativas boliviana, estadounidense, europea y recomendaciones de la OMS para la calidad del agua de consumo humano difieren en el número de compuestos contemplados así como en los valores límite establecidos. En la práctica, tales diferencias generan una desigual clasificación de la calidad de las muestras analizadas $\mathrm{y}$, por ende, una desigual estimación del número de personas expuestas a componentes del petróleo. Estas variaciones pueden condicionar en gran medida la toma de decisiones $\mathrm{y}$, en consecuencia, las intervenciones en gestión ambiental y en salud pública.

Hasta el momento existen muy pocos estudios que hayan realizado análisis de compuestos químicos derivados de petróleo en muestras de agua de consumo humano -o potencialmente consumible- y no se encontró ninguno que haya analizado varios compuestos simultáneamente.

Los compuestos contaminantes que se hallaron en mayor proporción en las muestras analizadas fueron los HTP, con concentraciones superiores a lo permitido por la norma boliviana en la mitad de las muestras (35). (Solo esta normativa establece valores límite para los HTP en el listado de sustancias para analizar en el agua potable.) Las concentraciones halladas en este estudio son similares a las encontradas tres años antes por Mamani y col. en la misma área geográfica, con una media de $1,3 \mu \mathrm{g} / \mathrm{L}(0-2880 \mu \mathrm{g} / \mathrm{L})$ en varios puntos examinados $(25,26)$.

La similitud de las concentraciones pese a la distancia temporal del muestreo podría sugerir la existencia de un vertido continuo desde las actividades de extracción a las fuentes de agua. Otro estudio, que analizó aguas consumibles o potencialmente consumibles en la amazonía ecuatoriana, también detectó concentraciones medias de $60,4 \mu \mathrm{g} / \mathrm{L}$ 
CUADRO 2. Distribución de la mediana de concentración de los contaminantes en el agua, según la fuente de la muestra, Bolivia, 2005

\begin{tabular}{|c|c|c|c|c|c|c|}
\hline \multirow{2}{*}{$\begin{array}{l}\text { Contaminante } \\
\text { (valor límite } \\
\text { norma boliviana }{ }^{a} \text { ) }\end{array}$} & \multicolumn{6}{|c|}{ Mediana [rango de concentración] ( $\mu \mathrm{g} / \mathrm{L})$} \\
\hline & Quebrada & Grifo & Río o arroyo & Pozo & Laguna & Total $(n=42)$ \\
\hline $\mathrm{HTP}^{\mathrm{b}}(10 \mu \mathrm{g} / \mathrm{L})$ & $50[<10-4330]$ & $540[<10-21010]$ & $10[<10-5730]$ & $10[<10-5140]$ & $<10$ & $50[<10-21010]$ \\
\hline $\begin{array}{l}\text { Benzo(a)pireno } \\
(0,2 \mu \mathrm{g} / \mathrm{L})\end{array}$ & $0[0-1,67]$ & $0[0-2,35]$ & 0 & 0 & 0 & {$[0-2,35]$} \\
\hline Benceno $(2 \mu \mathrm{g} / \mathrm{L})$ & $<1$ & $<1$ & $<1$ & $<1$ & $<1$ & $<1$ \\
\hline Tolueno $(700 \mu \mathrm{g} / \mathrm{L})$ & $<10$ & $<10$ & $<10$ & $<10$ & $<10$ & $<10$ \\
\hline Etilbenceno $(300 \mu \mathrm{g} / \mathrm{L})$ & $<10$ & $<10$ & $<10$ & $<10$ & $<10$ & $<10$ \\
\hline Xilenos $(500 \mu \mathrm{g} / \mathrm{L})$ & $<20$ & $<20$ & $<20$ & $<20$ & $<20$ & $<20$ \\
\hline Aluminio $(200 \mu \mathrm{g} / \mathrm{L})$ & $14,9[10-36]$ & $10[10-868]$ & $8698\left[7-3,09 \times 10^{4}\right]$ & $10[10-50,4]$ & 879 & 11 [7 - 30 868] \\
\hline Arsénico $(10 \mu \mathrm{g} / \mathrm{L})$ & 5,8 & $5,8[5,8-32]$ & $32[5,8-89]$ & 5,8 & 32 & $5,8[5,8-89]$ \\
\hline Bario $(700 \mu \mathrm{g} / \mathrm{L})$ & $189[27-388]$ & $136[18-199]$ & $139,5[38-230]$ & $27[20-106]$ & 166 & $151[18-388]$ \\
\hline Calcio $\left(2 \times 10^{5} \mu \mathrm{g} / \mathrm{L}\right)$ & $\begin{array}{c}3,32 \times 10^{4} \\
{\left[5,70 \times 10^{4}-7,35 \times 10^{4}\right]}\end{array}$ & $\begin{array}{c}5,22 \times 10^{4} \\
{\left[5,85 \times 10^{3}-2,79 \times 10^{5}\right]}\end{array}$ & $\begin{array}{c}2,85 \times 10^{4} \\
{\left[1,89 \times 10^{4}-4,90 \times 10^{4}\right]}\end{array}$ & $\begin{array}{c}7,80 \times 10^{4} \\
{\left[5,75 \times 10^{4}-2,30 \times 10^{5}\right]}\end{array}$ & $7,73 \times 10^{4}$ & $\begin{array}{c}4,83 \times 10^{4} \\
{\left[1-5,70 \times 10^{3}\right]}\end{array}$ \\
\hline Cadmio $(5 \mu \mathrm{g} / \mathrm{L})$ & 0,64 & $0,64[0,64-0,64]$ & $0,64[0,6-2,4]$ & $0,64[0,64-0,64]$ & 0,6 & $0,64[0,6-2,4]$ \\
\hline Cromo $(50 \mu \mathrm{g} / \mathrm{L})$ & 2,9 & $2,9[2,9-222]$ & $29,8[2,9-94]$ & 2,9 & 19 & $2,9[2,9-222]$ \\
\hline Cobre $(1000 \mu \mathrm{g} / \mathrm{L})$ & 17 & $17[4-17]$ & $20[8,7-36]$ & 17 & 20 & $17[4-36]$ \\
\hline Hierro (300 $\mu \mathrm{g} / \mathrm{L})$ & $18[6,8-74]$ & $9,1[1,5-417]$ & $942,1\left[19-3,09 \times 10^{4}\right]$ & $17[1,5-43]$ & 1,147 & $20\left[1,5-3,09 \times 10^{4}\right]$ \\
\hline Litio (2 $500 \mu \mathrm{g} / \mathrm{L})$ & 46 & $46[46-100]$ & $100[46-100]$ & $46[46-287]$ & 100 & $46[46-287]$ \\
\hline $\begin{array}{l}\text { Magnesio } \\
\left(1,5 \times 10^{5} \mu \mathrm{g} / \mathrm{L}\right)\end{array}$ & $\begin{array}{c}6,31 \times 10^{3} \\
{\left[1,79 \times 10^{3}-1,53 \times 10^{4}\right]}\end{array}$ & $\begin{array}{c}8,82 \times 10^{3} \\
{\left[1,76 \times 10^{3}-6,15 \times 10^{4}\right]}\end{array}$ & $\begin{array}{c}1,62 \times 10^{4} \\
{\left[6,44 \times 10^{3}-2,08 \times 10^{4}\right]}\end{array}$ & $\begin{array}{c}4,65 \times 10^{4} \\
{\left[1,20 \times 10^{4}-2,80 \times 10^{5}\right]}\end{array}$ & $6,35 \times 10^{4}$ & $\begin{array}{c}1,36 \times 10^{4} \\
{\left[1,76 \times 10^{3}-2,80 \times 10^{5}\right]}\end{array}$ \\
\hline Manganeso $(100 \mu \mathrm{g} / \mathrm{L})$ & $6[1,3-38]$ & $4[0,58-481]$ & $274[7,9-457]$ & $3[0,7-199]$ & 53 & $7,45[0,58-481]$ \\
\hline Sodio $\left(2 \times 10^{5} \mu \mathrm{g} / \mathrm{L}\right)$ & $\begin{array}{c}1,77 \times 10^{4} \\
{\left[6,53 \times 10^{3}-3,11 \times 10^{4}\right]}\end{array}$ & $\begin{array}{c}2,40 \times 10^{4} \\
{\left[6,42 \times 10^{3}-1,87 \times 10^{5}\right]}\end{array}$ & $\begin{array}{c}2,91 \times 10^{4} \\
{\left[2,25 \times 10^{4}-3,59 \times 10^{4}\right]}\end{array}$ & $\begin{array}{c}1,16 \times 10^{5} \\
{\left[2,29 \times 10^{4}-6,50 \times 10^{5}\right]}\end{array}$ & $4,71 \times 10^{4}$ & $\begin{array}{c}2,73 \times 10^{3} \\
{\left[6,42 \times 10^{3}-6,50 \times 10^{5}\right]}\end{array}$ \\
\hline Níquel (50 $\mu \mathrm{g} / \mathrm{L})$ & 10 & $10[10-840]$ & $10[5,7-183]$ & 10 & 5,7 & $10[5,7-840]$ \\
\hline Zinc (5 $000 \mu \mathrm{g} / \mathrm{L})$ & $67[52-67]$ & $67[52-216]$ & $115,5[33-413]$ & 67 & 87 & $67[33-413]$ \\
\hline
\end{tabular}

Fuente: elaboración de los autores a partir de resultados de laboratorio.

a Datos basados en la referencia 35.

${ }^{\mathrm{b}}$ Hidrocarburos totales de petróleo.

(0-2 $883 \mu \mathrm{g} / \mathrm{L})$ en ríos cercanos a pozos de extracción de petróleo, similares a las del presente trabajo (39). En el río Vatinsky Egan (Siberia occidental), en muestras tomadas donde atraviesa un campo petrolífero, se hallaron concentraciones ligeramente inferiores, de 43-85 $\mu \mathrm{g} / \mathrm{L}(40)$. Hay que tener en cuenta que en aguas superficiales, no aptas en principio para consumo humano, generalmente las concentraciones halladas de HTP están en un rango de entre 0,99 $\mu \mathrm{g} / \mathrm{L}$ y $200 \mu \mathrm{g} / \mathrm{L}(41,42)$, muy similares a las del presente estudio.

Un tercio de las muestras analizadas, principalmente las de grifo, revelaron HAP en concentraciones que superan el valor límite establecido por la normativa europea (36). Aunque la fuente principal de los HAP es la combustión incompleta de combustibles fósiles, estos compuestos pueden provenir también de otras fuentes. Un informe de la OMS advierte que en las zonas urbanas el rango de concentración de los 16 HAP en agua potable oscila entre 0,001 y 0,011 $\mu \mathrm{g} / \mathrm{L}$ (43). En zonas rurales, la combustión no suele ser la principal fuente de contaminación de este tipo de compuestos debido a la inexistencia de focos emisores. Análisis realizados en aguas superficiales no potables de zonas con industria petrolífera documentaron concentraciones similares a las del presente trabajo. En los ríos Uruguay y Río de la Plata se detectaron concentraciones de HAP de entre 1,8 y $12 \mu \mathrm{g} / \mathrm{L}$, en aguas costeras de la Patagonia, afectadas por actividades de extracción de petróleo, el rango fue de 8-41 $\mu \mathrm{g} / \mathrm{L}(44)$, y en el York River (Estados Unidos), próximo a una refinería de petróleo, las concentraciones se situaron en el rango de 2,09 a 122,85 $\mu \mathrm{g} / \mathrm{L}$ (45). La semejanza de las concentraciones halladas en el presente estudio con las de otros trabajos realizados en diversas zonas petrolíferas parece apoyar la hipótesis de que esa contaminación tiene su fuente en las descargas o derrames provocados por las actividades petrolíferas que se llevan a cabo en dichas zonas.

Las concentraciones de BTEX en las muestras analizadas estuvieron por debajo de los límites establecidos en las normativas aplicadas en el estudio. A este respecto, es importante tener en cuenta que la volatilidad de estos compuestos podría impedir su identificación en concentraciones superiores a unos pocos $\mu \mathrm{g} / \mathrm{L}$ en la superficie de las aguas (46).
En el caso de los metales, las muestras revelaron concentraciones de aluminio, arsénico, cromo, hierro, manganeso, sodio y níquel superiores a las permitidas por las distintas normativas de referencia. Investigaciones previas en la zona del estudio han localizado pequeños depósitos de minerales metálicos, pero dado que hasta la fecha no han sido explotados es bastante improbable que constituyan la fuente de la presencia de metales en el agua analizada.

Sí está documentada la contaminación del río Pilcomayo por metales derivados de las operaciones mineras de Potosí, al suroeste del país, aunque la influencia de estas actividades en la zona del presente estudio debería ser limitada (47-51). En 2001, Hudson-Edwards y col. observaron que la presencia de estos metales iba reduciéndose aguas abajo, revelando concentraciones semejantes a las notificadas en la zona preminera de la región de Villamontes y menores a las encontradas en el presente estudio (48).

Tales hallazgos parecen indicar que la industria del petróleo en el Chaco Boliviano es en efecto la principal fuente de aporte de metales al agua de la zona, más aún por su coherencia con las con- 
FIGURA 2. Distribución de las muestras de agua contaminadas por uno o más compuestos en cada una de las comunidades estudiadas y para cada una de las fuentes, según las normativas boliviana, europea y estadounidense, así como las de la OMS, Bolivia, 2005

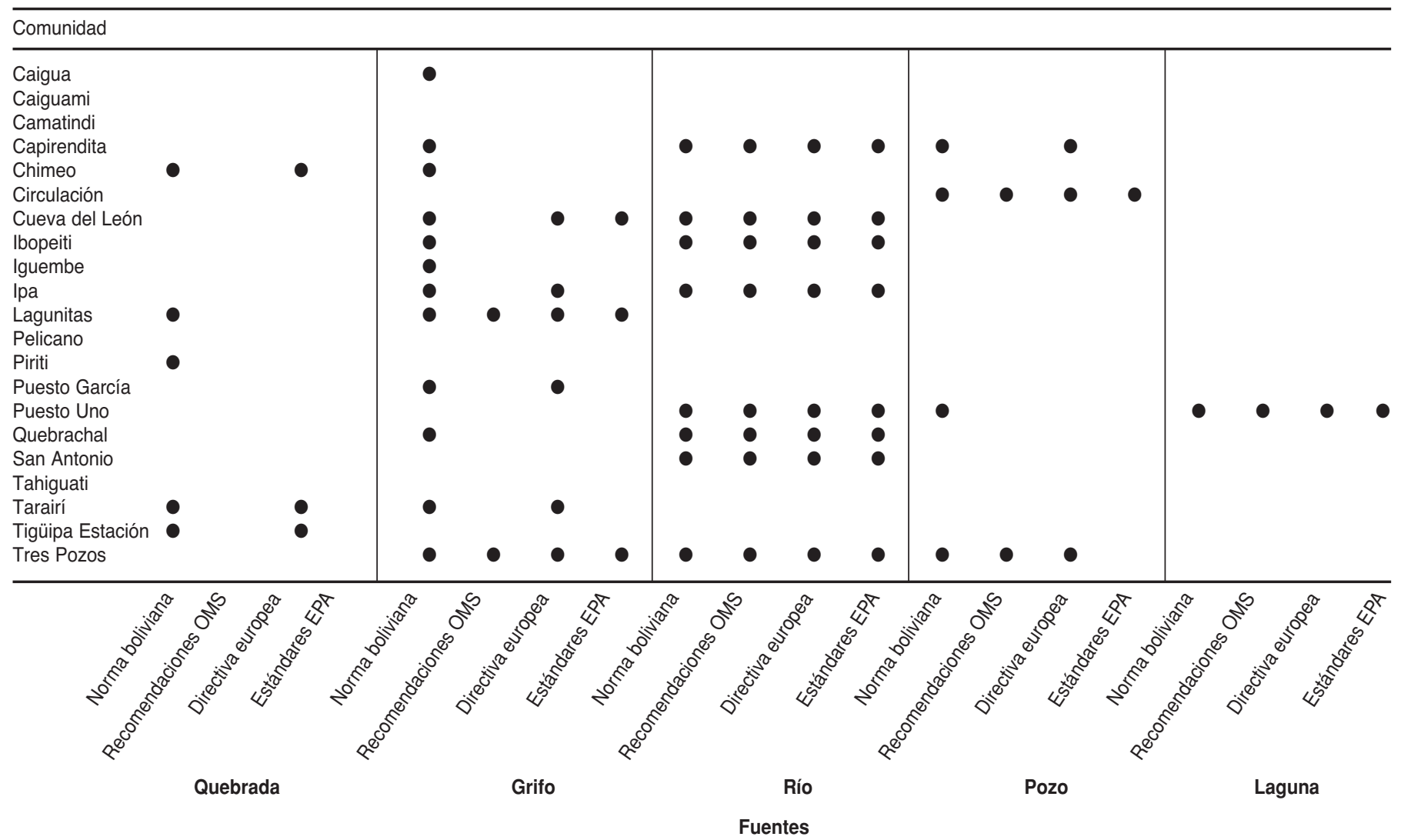

Fuente: elaborada por los autores con base en las referencias 35 a 38.

Nota: Abreviaturas. EPA: Agencia para la Protección del Medio Ambiente (Estados Unidos); OMS: Organización Mundial de la Salud.

centraciones de metales encontradas en otras zonas con actividades petrolíferas, y particularmente en las muestras del presente estudio no relacionadas con las aguas del Pilcomayo. Asimismo, análisis de agua potencialmente consumible a su paso por los campos petroleros de Siberia occidental detectaron concentraciones de magnesio (2 400-13 400) $\mu \mathrm{g} / \mathrm{L}$, calcio (6 000-180 000) $\mu \mathrm{g} / \mathrm{L}$ y hierro (100$6800) \mu \mathrm{g} / \mathrm{L}(40)$. Otro estudio en aguas superficiales no consumibles en Egipto encontró vanadio $(3,62-8,89) \mu \mathrm{g} / \mathrm{L}$, níquel $(1,30-3,72) \mu \mathrm{g} / \mathrm{L}$, magnesio (78-153) $\mu \mathrm{g} / \mathrm{L}$, hierro $(1,40-4,93) \mu \mathrm{g} / \mathrm{L}$, cobre $(0,01-0,04) \mu \mathrm{g} / \mathrm{L}$ y zinc $(0,09-0,13) \mu \mathrm{g} / \mathrm{L}$, como puede verse, en rangos de concentraciones muy por encima a las halladas en este trabajo (52).

La principal limitación del presente estudio ha sido la utilización de un muestreo puntual y discreto para analizar la calidad de las fuentes de agua de consumo que, al no tener en cuenta variaciones estacionales de concentración de los compuestos, podría haber llevado a sobreestimar o subestimar el grado de exposición real. Aplicando un criterio conservador, se decidió realizar el muestreo durante la estación seca, cuando se esperan las mayores concentraciones de los diferentes contaminantes, bajo el supuesto de que en este período se alcanzarían las máximas concentraciones a las que podrían estar expuestos los potenciales consumidores.

Cabe mencionar que si la toma de la muestra ocurre tras la desaparición de los compuestos pueden no detectarse elevaciones de la concentración debidas a vertidos puntuales, así como las exposiciones agudas e intensas asociadas, bien por desplazamiento del vertido aguas abajo o por dilución del compuesto con los nuevos aportes de agua. Esta limitación solamente es salvable con redes de control de calidad de las aguas que permitan tomar muestras compuestas y diarias, un escenario deseable pero difícilmente alcanzable en el área de es- tudio. Finalmente, las concentraciones halladas de selenio y plomo quedaron fuera del rango de detección de la técnica por un error en la elección de los límites de la recta de calibración en el laboratorio externo encargado de realizar los análisis. Por ello, en análisis de los datos se excluyó a ambos metales.

\section{Conclusiones y recomendaciones}

Los análisis de agua, realizados en las fuentes de agua para consumo humano en comunidades situadas a menos de $30 \mathrm{~km}$ de campos de extracción de petróleo, detectaron concentraciones de HTP, HAP y metales muy por encima de los límites máximos establecidos por las normativas boliviana, europea y estadounidense, así como de los recomendados por la OMS.

Si bien el estudio no incluyó la evaluación de los efectos que dichas exposiciones pueden tener sobre la salud de las poblaciones que consumen el agua anali- 
FIGURA 3. Porcentajes de muestras contaminadas por tipo de compuestos de petróleo analizados y fuentes de consumo según las diferentes normativas y recomendaciones utilizadas en el estudio, Bolivia, 2005

Legislación boliviana

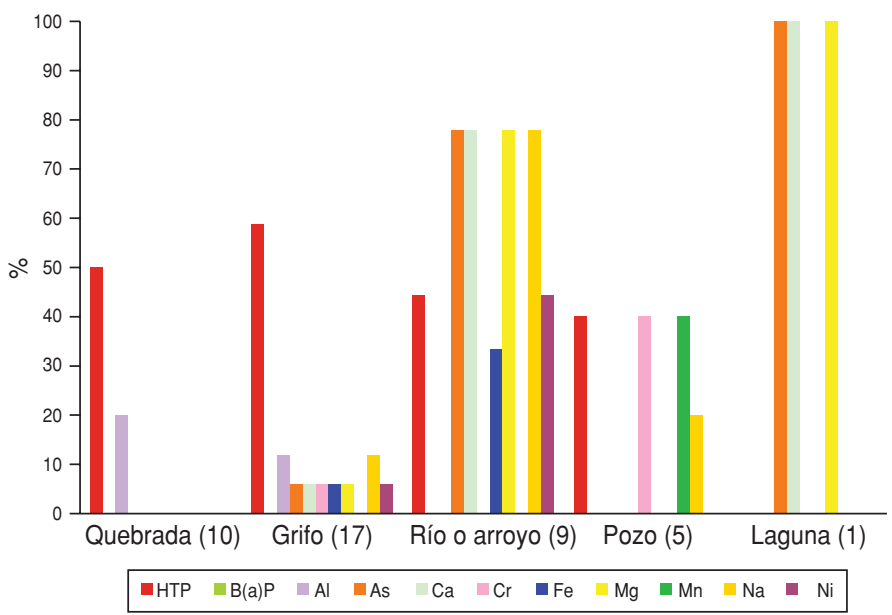

Estándares EPA

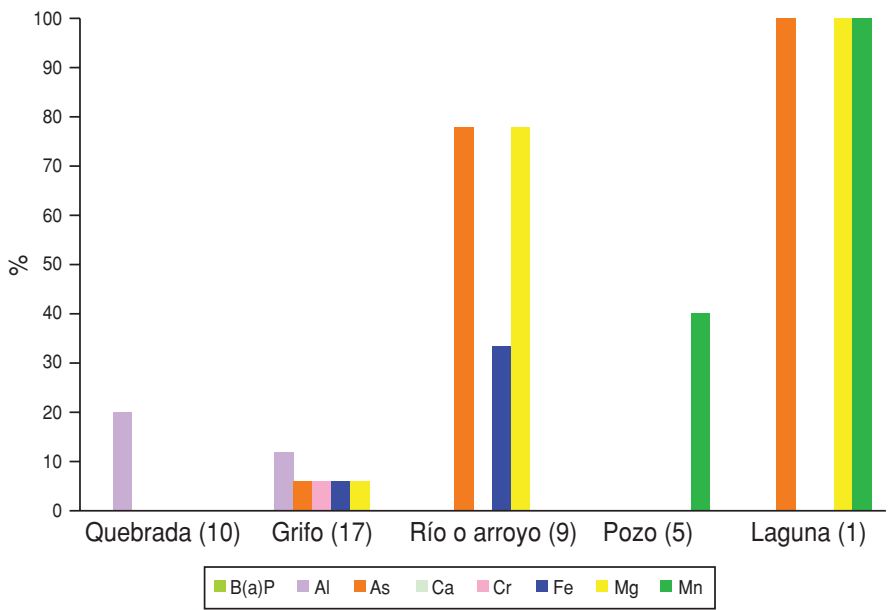

Legislación europea

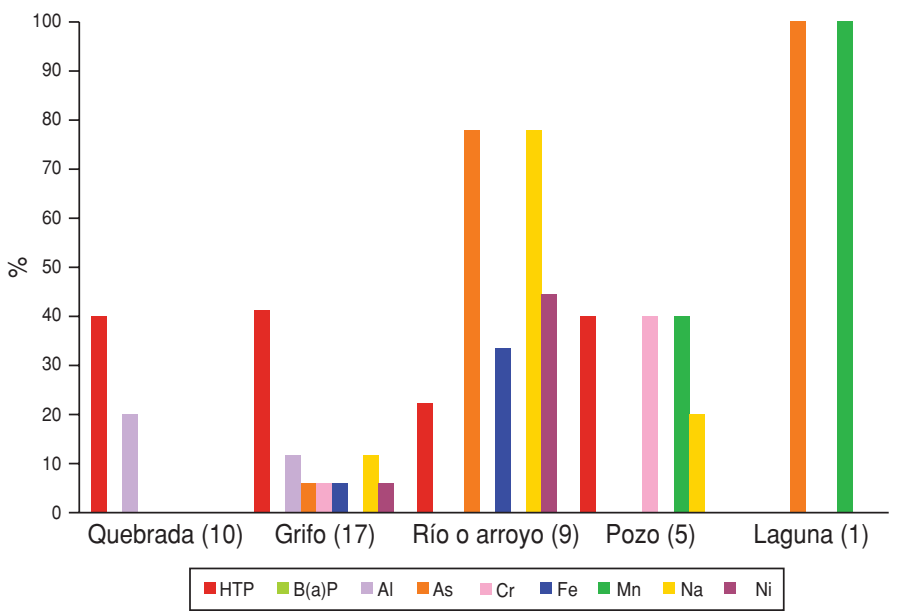

Recomendaciones de la OMS

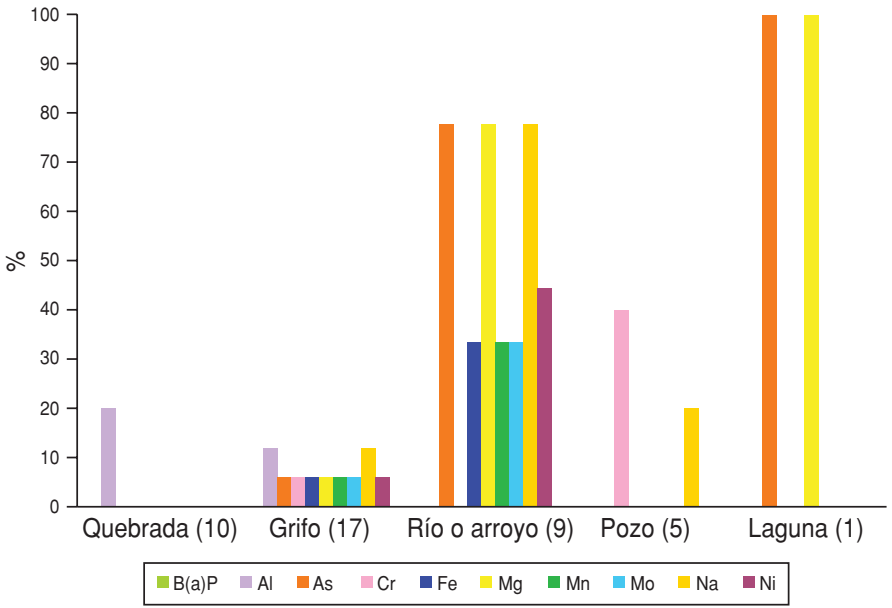

Fuente: elaborada por los autores con base en las referencias 35 a 38.

Nota: Abreviaturas. EPA: Agencia para la Protección del Medio Ambiente (Estados Unidos); OMS: Organización Mundial de la Salud.

zada, cabe señalar que ciertos componentes del petróleo son considerados carcinogénicos potenciales por la Agencia Internacional para la Investigación sobre el Cáncer, la EPA y el Consejo Asesor para las Ciencias (53). Es más, dado que los riesgos asociados con la exposición a concentraciones similares $\mathrm{o}$ incluso inferiores a las encontradas en este trabajo han sido ampliamente documentados en investigaciones previas, no parece muy arriesgado pensar que las comunidades aquí estudiadas ya podrían estar sufriendo tales efectos en su salud $(4,5,9,12,17,54)$.

Por último, a partir de los resultados obtenidos en el estudio se ofrecen las siguientes recomendaciones:
- Establecer medidas ambientales que permitan monitorear sistemáticamente el grado de contaminación del agua de consumo de las comunidades que residen en zonas cercanas a actividades de extracción de petróleo.

- Exigir a las empresas el cumplimiento de las normativas en vigor relativas a los vertidos de la actividad industrial desarrollada. Estas normativas deberían responder a la realidad local pues, como pone en evidencia el presente estudio, las recomendaciones dirigidas a otros países a menudo no incluyen niveles de referencia para compuestos que no son prioritarios en el país que las emite, quizá por lo improbable de su presencia en el agua potable. Un ejemplo de esto es la no inclusión de HTP y HAP en la normativa de la EPA en Estados Unidos.

- Realizar estudios prospectivos que permitan analizar la evolución de la concentración en el tiempo $\mathrm{y}$, por tanto, detectar las fluctuaciones asociadas a fugas puntuales y variaciones estacionales.

- A la importante medición del grado de exposición de estas comunidades a los compuestos del petróleo, es prioritario sumar el análisis de su impacto real en la salud de esas poblaciones mediante estudios prospectivos diseñados al efecto. 


\section{REFERENCIAS}

1. Instituto Nacional de Estadística, Bolivia. Inversión Extranjera Directa (1996-2002). Hallado en: http://www.ine.gov.bo/pdf/ied/ ied.pdf. Acceso el 05 de agosto de 2010.

2. Hawley G. The Condensed Chemical Dictionary. 14th ed. New York: John Wiley \& Sons; 2001.

3. Rajput N, Khemani LD, Lakhani A. Polycyclic aromatic hydrocarbons in ambient air at Agra: distribution and toxicity assessment. J Environ Sci Eng. 2008;50(2):111-4.

4. San Sebastián M, Hurtig AK. Oil exploitation in the Amazon basin of Ecuador: A public health emergency. Rev Panam Salud Publica. 2004;15(3):205-11.

5. San Sebastián M, Armstrong B, Stephens C. La salud de mujeres que viven cerca de pozos y estaciones de petróleo en la Amazonía ecuatoriana. Rev Panam Salud Publica. 2001;9(6): 375-84.

6. Wichmann FA, Muller A, Busi LE, Cianni N, Massolo L, Schlink U, et al. Increased asthma and respiratory symptoms in children exposed to petrochemical pollution. J Allergy Clin Immunol. 2009;123(3):632-8.

7. Gazdek D, Strnad M, Mustajbegovic J, NemetLojan Z. Lymphohematopoietic Malignancies and Oil Exploitation in Koprivnica-Krizevci County, Croatia. Int J Occup Environ Health. 2007;13:258-67.

8. Yu CL, Wang SF, Pan PC, Wu MT, Ho CK, Smith TJ, et al. Residential exposure to petrochemicals and the risk of leukemia: using geographic information system tools to estimate individual-level residential exposure. Am J Epidemiol. 2006;164(3):200-7.

9. Hurtig AK, San Sebastián M. Incidence of childhood leukemia and oil exploitation in the Amazon basin of Ecuador. Int J Occup Environ Health. 2004;10(3):245-50.

10. Weng H, Tsai SS, Chiu HF, Yang CY, Yu CL. Association of childhood leukemia with residential exposure to petrochemical air pollution in Taiwan. Inhal Toxicol. 2008;20(1):31-6.

11. Liu CC, Chen CC, Wu TN, Yang CY. Association of brain cancer with residential exposure to petrochemical air pollution in Taiwan. J Toxicol Environ Health A. 2008;71(5):310-4.

12. Hurtig AK, San SM. Geographical differences in cancer incidence in the Amazon basin of Ecuador in relation to residence near oil fields. Int J Epidemiol. 2002;31(5):1021-7.

13. Yang CY, Cheng BH, Hsu TY, Tsai SS, Hung $\mathrm{CF}, \mathrm{Wu} \mathrm{TN}$. Female lung cancer mortality and sex ratios at birth near a petroleum refinery plant. Environ Res. 2000;83:33-40.

14. Lin MC, Chiu HF, Yu HS, Tsai SS, Cheng BH, $\mathrm{Wu} \mathrm{TN}$, et al. Increased risk of preterm delivery in areas with air pollution from a petroleum refinery plant in Taiwan. J Toxicol Environ Health A. 2001;64(8):637-44.

15. Tsai SS, Yu HS, Liu CC, Yang CY. Increased incidence of preterm delivery in mothers residing in an industrialized area in Taiwan. J Toxicol Environ Health A. 2003;66(11):987-94.

16. Yang CY, Chang CC, Chuang HY, Ho CK, Wu TN, Chang PY. Increased risk of preterm delivery among people living near the three oil refineries in Taiwan. Environ Int. 2004;30(3): $337-42$.
17. San Sebastián M, Armstrong B, Stephens C. Outcomes of pregnancy among women living in the proximity of oil fields in the Amazon basin of Ecuador. Int J Occup Environ Health. 2002;8(4):312-9.

18. Lin CM, Lin CY, Mao IF. Increased risk of term low-birth-weight infants in a petrochemical industrial city with high air pollution. Arch Environ Health. 2004;59(12):663-8.

19. Ortiz P, Varea A. La explotación petrolera en el Ecuador: historia e impactos socioambientales. Marea negra en la Amazonía: conflictos socioambientales vinculados a la actividad petrolera en el Ecuador. Quito: Abya-Yala; 1995. Pp. 71-88.

20. Center for Economic and Social Rights (CESR). Rights Violations in the Ecuadorian Amazon: The Human Consequences of Oil Development. Health Hum Rights. 1994;1(1): 82-100.

21. Herbas G. Explotación de hidrocarburos y el medio ambiente en un escenario neoliberal Derrame de Transredes (Enron/Shell) en el río Desaguadero. Hallado en: http://www. ibcperu.org/doc/isis/8654.pdf. Acceso el 10 de octubre de 2010

22. Gavaldá M. Las Manchas del Petróleo Boliviano. Cochabamba, Bolivia: CEDIB; 1999.

23. FOBOMADE. El caso del gasoducto BoliviaBrasil. Hallado en: http://www.ibcperu.org/ doc/isis/8653.pdf. Acceso el 19 de octubre de 2010.

24. Molina P. Relaciones energéticas, integración y medio ambiente. El caso del gasoducto Bolivia-Brasil. En: Relaciones Energéticas Bolivia-Brasil. La Paz: Foro Boliviano Medio Ambiente y Desarrollo; 2005. Pp. 27-40. Hallado en: http://www.fobomade.org.bo/ documentos/libros/energ_bol_bras.pdf. Acceso el 19 de octubre de 2010.

25. Mamani Q, Suárez N, García C. Estudio socioambiental de la contaminación del agua por actividad hidrocarburífera en la Serranía Aguaragüe. Zona de influencia Villa Montes. Tarija, Bolivia: Fundación PIEB; 2002.

26. Mamani Q, Suárez N, García C. Contaminación del agua e impactos por la actividad hidrocarburífera en Aguaragüe. Tarija, Bolivia: Fundación PIEB, Dirección de Investigación Científica y Tecnológica U.A.J.M.S., Centro Eclesial de Documentación, Centro de Estudios Regionales para el Desarrollo de Tarija (La Paz); 2003.

27. Mediano C. El impacto de la extracción petrolera en la salud: Una experiencia en Bolivia. Madrid: medicusmundi; 2004.

28. Instituto Nacional de Estadística, Bolivia. Bolivia: procedencia y distribución del agua para beber y cocinar en la vivienda de los hogares, censos de 1992 y 2001. Hallado en: http://www.ine.gov.bo/indice/visualizador. aspx?ah=PC3030312.HTM. Acceso el 06 de agosto de 2010.

29. Santos Guzmán J, Guerrero Medrano L, Reyna Caamaño R, Mejía Velásquez G. Marco legislativo del suministro de agua en México. Rev Panam Salud Publica. 2009;26(6):549-52.

30. Bernal F. Petróleo, Estado y Soberanía: hacia la empresa multiestatal latinoamericana de hidrocarburos. Buenos Aires: Biblos; 2005.
31. International Organization for Standardization (ISO). Guidance on the design of sampling programmes, ISO 5667-1:1980.

32. International Organization for Standardization (ISO). Guidance on sampling techniques, ISO 5667-2:1991.

33. International Organization for Standardization (ISO). Guidance on the preservation and handling of water samples, ISO 5667-3:2003.

34. Clesceri LS, Greenberg AE, Eaton AD. Standard Methods for the Examination of Water and Wastewater. 20th ed. APHA/AWWA/ WPCF. Washington, DC: United Book; 1998.

35. Ministerio de Servicios y Obras Públicas, Bolivia. Reglamento nacional para el control de la calidad del agua para consumo humano NB 512 (Enero 2005).

36. Comunidad Europea. Directiva 98/83/EC del Consejo Europeo, relativa a la calidad de las aguas destinadas al consumo humano (3 de noviembre de 1998). Diario Oficial no. L 330 de 5.12.1998. Pp. 32-54.

37. U.S. Environmental Protection Agency (EPA). Current Drinking Water Standards: National Primary and Secondary Drinking Water Regulations. Washington, DC: U.S.-EPA Office of Ground Water and Drinking Water; 1999. Hallado en: http:/ / www.epa.gov/safewater/ contaminants/index.html. Acceso el 11 de agosto de 2010

38. Word Health Organization (WHO). Guidelines for drinking-water quality. 3rd ed. Geneva: WHO; 2008.

39 San Sebastián M. Informe Yana Curi: Impacto de la actividad petrolera en la salud de poblaciones rurales de la Amazonía ecuatoriana. Barcelona: Icaria; 2000

40. Moskovchenko DV, Babushkin AG, Artamonova GN. Surface water quality assessment of the Vatinsky Egan River catchment, West Siberia. Environ Monit Assess. 2009;148(1-4):359-68.

41. Al-Imarah FJ. Petroleum hydrocarbons in water and sediments of northwest Arabian Gulf 1980-2005. Aquatic Ecosystem Health \& Management. 2007;10(3):335-40.

42. Douabul AAZ, Al-Saad HT. Seasonal variations of oil residues in water of Shatt al-Arab River, Iraq. Water Air Soil Pollut. 1985;24: 237-46.

43. World Health Organization (WHO). Polynuclear aromatic hydrocarbons in Drinkingwater. Background document for development of WHO Guidelines for Drinking-water Quality. Geneva: WHO; 2003.

44. Barra R, Castillo C, Machado JP. Polycyclic Aromatic Hydrocarbons in the Sourth American Environmental. Rev Environ Contam Toxicol. 2007;191:1-22

45. Countway RE, Dickhut RM, Canuel EA. Polycyclic aromatic hydrocarbon (PAH) distributions and associations with organic matter in surface waters of the York River, VA Estuary. Estuary Org Geochem. 2003;34:209-24.

46. Karaconji B, Skender L, Karacic V. Benzene, toluene, ethylbenzene, and isomeric xylenes in various water samples in Croatia. Bull Environ Contam Toxicol. 2006;76(3):458-62.

47. Garcia-Guinea J, Huascar M. Mining waste poisons river basin. Nature. 1997;387(6629):118. 
48. Hudson-Edwards K, Macklin M, Miller J, Lechler P. Sources, distribution and storage of heavy metals in the Río Pilcomayo, Bolivia. J Geochem Explor. 2001;72:229-50.

49. Macklin M, Payne I, Preston D, Sedgwick C. Review of the Porco mine tailings dam burst and associated mining waste problems, Pilcomayo basin, Bolivia. UK: Report to the UK Overseas Development Agency; 1996.

50. Miller JR, Hudson-Edwards KA, Lechler PJ, Preston D, Macklin MG. Heavy metal contamination of water, soil and produce within riverine communities of the Rio Pilcomayo basin, Bolivia. Sci Total Environ. 2004;320 (2-3):189-209.

51. Smolders A, Guerrero Hiza M, Van der Velde G, Roelfofs J. Dynamics of discharge, sediment transport, heavy metal pollution and sábalo (Prochilodus lineatus) catches in the lower Pilcomayo river (Bolivia). River Research and Applications. 2002;18(5):415-27.

52. Shimy TM. Distribution of seleted trace metals and its relation with environmental pollution. Energy Sources. 1997;19:851-60.
53. Yosie TF. The petroleum industry and the public health debate over petroleum products. J Expo Anal Environ Epidemiol. 1992; 2(1):1-3.

54. San Sebastián M, Armstrong B, Cordoba JA Stephens C. Exposures and cancer incidence near oil fields in the Amazon basin of Ecuador. Occup Environ Med. 2001;58(8):517-22.

Manuscrito recibido el 15 de febrero de 2010. Aceptado para publicación, tras revisión, el 15 de julio de 2010.

ABSTRACT Objective. To determine the concentrations of petrochemical compounds in the drinking water sources of communities located near oil-producing fields in the Bolivian Chaco region.

Water pollution in sources close to oil-producing fields of Bolivia

Key words
Methods. Data were collected on total petroleum hydrocarbons (TPH), 16 polycyclic aromatic hydrocarbons (PAH), including benzene, toluene, ethylbenzene, and xylenes (BTEX), and 22 metals in samples from 42 sources of water for human consumption located less than $30 \mathrm{~km}$ from an oil-producing field. Distribution of the concentration and adherence to the standards contained in the Bolivian, European, and United States regulations, as well as the recommendations of the World Health Organization, were analyzed.

Results. In $76.19 \%$ of the samples, some petrochemical contaminant was found in concentrations higher than permissible in any of the four sets of regulations mentioned. The water samples with the highest contamination levels were from faucets and rivers. The most common contaminants were $\mathrm{TPH}, \mathrm{PAH}$, aluminum, arsenic, manganese, and iron.

Conclusions. Communities within a $30 \mathrm{~km}$ radius of the oil-producing fields in the Bolivian Chaco region consume water with TPH, PAH, and metal concentrations well above the levels permitted in the Bolivian regulations and international standards, putting the public health of their residents at serious risk.

Water pollution; water pollutants; public standposts; potable water; chemical contamination; river pollution; hydrocarbons; petroleum; environmental health; Bolivia. 